\title{
Weighted Tradeoff Between Spectral Efficiency and Energy Efficiency in Energy Harvesting Systems
}

\author{
Arooj Mubashara Siddiqui ${ }^{1}$, Leila Musavian² ${ }^{2}$ Sonia Aïssa ${ }^{3}$ and Qiang $\mathrm{Ni}^{4}$ \\ ${ }^{1}$ 5GIC, ICS, University of Surrey, UK, email: a.siddiqui@ surrey.ac.uk \\ ${ }^{2}$ CSEE, University of Essex, UK, email: leila.musavian@essex.ac.uk \\ ${ }^{3}$ INRS-EMT, University of Quebec, Montreal, QC, Canada, email: aissa@emt.inrs.ca \\ ${ }^{4}$ SCC, InfoLab 21, Lancaster University, UK, email: q.ni@lancaster.ac.uk
}

\begin{abstract}
This paper proposes a new power allocation scheme to jointly optimize energy efficiency (EE) and spectral efficiency (SE) of a point-to-point communication system in which the transmitter is equipped with fixed as well as energy harvesting batteries. Time switching protocol is used such that in each time frame the node either harvests energy or transmits information. Firstly, a multi-objective optimization problem which jointly optimizes EE and SE is formulated. An importance weight parameter is introduced to control the priority level between EE and SE. Secondly, the multi-objective problem is transformed into a singleobjective optimization problem by using importance weight, and then solved through fractional programming. Using the KarushKuhn-Tucker conditions, the optimum power allocation scheme without input power constraint is developed. The ensuing solution is then generalized for system operation with average input power constraint. Closed-form expressions are derived and tested through simulations. Numerical results results are provided, and show the impact of the harvested power in improving the overall rate of the system. Also investigation is done to analyze the effect of system parameters on the achievable trade-off performance of the energy-harvesting based system.

Index Terms-Energy harvesting, energy efficiency, spectral efficiency, multi-objective optimization, power allocation.
\end{abstract}

\section{INTRODUCTION}

The fast growing demand for higher data rates requires more energy consumption, which translates into infrastructures with higher energy costs and higher impact on air pollution. In addition, there is a huge gap between the technological advancements for satisfying the increasing rate demands and those for battery life improvements. Energy harvesting (EH) techniques promise environmental friendly solutions to the increasing demand for energy [1]. In fact, harvesting energy through multiple resources gained significant attention in recent times, where practical devices, e.g., Power-cast power-harvester devices, convert the radio waves into direct-current (DC) power in order to increase the life-cycle of devices with low-power consumption [2].

Energy efficiency (EE), defined as the bit per hertz per unit of energy consumption, and spectral efficiency (SE) which is one of the key performance indicators of the Third Generation Partnership Project (3GPP) evolution, are two major performance metrics in wireless communication systems that are hard to balance [3]. Many of the traditional approaches aiming at improving one of these metrics result in significant deterioration of the other. In fact, since EE and SE do not always coincide and rather conflict most of the times, balancing the achievable EE-SE is critical [4], [5]. Hence, research interests have been shifted towards studying EH technologies which promise to provide a reliable approach to improve EE while maintaining SE [6].

In the past few years, there has been a significant shift in research on EH communications with focus on developing EH protocols that improve the rate and EE of communication systems [7]. In this regard, the maximum achievable energy and spectral efficiencies in downlink orthogonal frequency division multiple access (OFDMA) networks are investigated in [8]. Generally, most wireless communication systems require more than one quality-of-service (QoS) requirement to be maintained [9]. Solution to such optimization problems, which involve more than one QoS requirement, can be obtained by solving a multi-objective optimization problem (MOP) [10]. In [11], the EE-SE maximization problem of a wireless EHbased cooperative communication system with best relay selection scheme was modeled into a MOP, and then transformed into a single-objective optimization problem (SOP) by using a weighted sum method. Knowing the imperfect channel estimation, the inverse of EE and SE are combined in a weighted optimization problem, as discussed in [12]. A MOP approach to maximize the throughput of a cognitive radio network with multiple secondary users using interference efficiency with ergodic sum-rate of the cognitive users is described in [13]. Optimizing the SE-EE tradeoff with green energy evaluation framework for wireless backhaul heterogeneous networks by jointing the load balancing and interference management is studied in [14]. Energy-spectral efficiency tradeoff in simultaneous wireless information and power transfer system is presented in [15].

Despite the major benefits that EH technology can have on the life and the performance of battery-limited communication 
devices, the majority of the research works in the literature is focused on transmitting devices relying only on energy harvested from external sources, with no consideration of energy from fixed batteries [16]. Generally, it is inevitable that some sort of limited fixed battery is implemented within the nodes of the communication system [17]. However, to the authors' best knowledge, there has been no work in the literature which deals with EH-MOP in a system with fixed as well as harvesting batteries. Detailed research is required to analyze how to balance EE and SE with EH battery.

In this paper, we investigate and discuss the impact of $\mathrm{EH}$ on balancing the achievable EE and SE so as to improve the overall system performance of point-to-point communication. The contributions of the paper are listed below.

- We formulate the achievable EE and SE when the transmitter has an EH battery in addition to the limited fixed battery. The purpose is to obtain an optimal transmit power allocation strategy to jointly maximize these two contrasting metrics.

- A MOP is formulated with the aim to jointly optimize EE and SE. The priority level of EE and SE can be varied by introducing an importance weight factor. The MOP is further converted into a SOP by using importance weight, and then solved using fractional programming.

- We provide closed-form expressions for the solution of the formulated SOP. The impact of the EH time-switching parameter, importance weight, circuit powers and harvested energy level on the achievable trade-off performance is investigated and also analyzed through Monte-Carlo simulations. Guidelines on how to choose the time-switching parameter and importance weight to benefit either EE or SE are also provided.

\section{System Model}

We consider point-to-point communication over wireless fading channel. The channel follows a block fading model, i.e., channel gain is invariant during each fading block, but varies independently from one block to another. The length of each fading block is denoted by $T_{\text {block}}$. The symbol duration is given by $T_{\mathrm{s}}=1 / B$, where $B$ is the system bandwidth. Furthermore, the noise is considered as additive white Gaussian noise. The channel state information (CSI) is estimated at the receiver, and is assumed to be fed back to the transmitter through an error-free feedback channel.

In this system, the transmitter is equipped with a fixed battery as well as an EH battery. The harvest-use approach is used, in which the harvested energy cannot be stored and should be used as soon as it becomes available [18]. The proposed model can replenish energy from different sources. Here, the harvesting relies on solar energy. The device either harvests energy or transmits data. Therefore, the time-switching (TS) approach is used at the transmitter, so that the EH takes place during a fraction $(\tau)$ of the time frame, and the information communication occupies the remaining $(1-\tau)$ fraction of the time frame.

\section{A. Spectral Efficiency}

The SE of the system, in $\mathrm{b} / \mathrm{s} / \mathrm{Hz}$ [7], is given by

$\mathrm{SE}=(1-\tau) \mathbb{E}\left[\log _{2}\left(1+\left(P_{\mathrm{t}}(\gamma)+\frac{e_{\mathrm{h}} \tau}{1-\tau}\right) \frac{\gamma}{M_{\mathcal{L}}}\right)\right]$,

where $P_{\mathrm{t}}(\gamma)$ is the instantaneous transmission power from the fixed battery expressed as a function of the channel power gain $\gamma^{1}, e_{\mathrm{h}}$ is the power from the harvesting battery, ${ }^{2} \mathbb{E}_{\gamma}[$.$] is the$ expectation operator over the channel power gain $\gamma$, and $M_{\mathcal{L}}=$ $P_{\mathrm{L}} N_{\mathrm{o}} B$, where $P_{\mathrm{L}}$ denotes the path-loss and $N_{\mathrm{o}}$ indicates the noise power density ${ }^{3}$. Assuming unit variance Rayleigh fading, the probability density function of $\gamma$ is $f_{\gamma}(\gamma)=e^{-\gamma}$.

\section{B. Energy Efficiency}

We formulate the $\mathrm{EE}$ as the ratio of the information rate to the sum of the transmission power and the total circuit power. The sum power is given by

$$
P_{\text {total }}(\gamma)=P_{\mathrm{c} e} \tau T_{\mathrm{s}}+(1-\tau) T_{\mathrm{s}}\left(P_{\mathrm{c}}+\frac{1}{\varepsilon} \mathbb{E}_{\gamma}\left[P_{\mathrm{t}}(\gamma)\right]\right),
$$

where $P_{\mathrm{c}}$ is the information processing circuit power [18], $0 \leq \varepsilon \leq 1$ is the power amplifier efficiency, and $P_{\mathrm{c} e}$ is the circuit power during the harvesting time $\tau T_{\mathrm{s}}$. Therefore, the total achievable EE, in $\mathrm{b} / \mathrm{J} / \mathrm{Hz}$, can be expressed as

$$
\mathrm{EE}=\frac{(1-\tau) \mathbb{E}\left[\log _{2}\left(1+\left(P_{\mathrm{t}}(\gamma)+\frac{e_{\mathrm{h}} \tau}{1-\tau}\right) \frac{\gamma}{M_{\mathcal{L}}}\right)\right]}{P_{\text {total }}(\gamma)} .
$$

\section{Optimal Power Allocation}

We first formulate the EE-SE trade-off as a MOP with average input power constraint:

$$
\begin{aligned}
& \max _{P_{\mathrm{r}}(\gamma) \geq 0} \text { EE } \quad \text { and } \max _{P_{\mathrm{r}}(\gamma) \geq 0} \mathrm{SE} \\
& \text { subject to: } \quad \mathbb{E}_{\gamma}\left[P_{\mathrm{r}}(\gamma)\right] \leq \frac{P_{\max }}{M_{\mathcal{L}}}
\end{aligned}
$$

where $P_{\max }$ is the average transmission power limit from the fixed battery.

Now, we introduce scaled notation for achievable EE as $\mathrm{EE}_{\mathrm{r}}=\mathrm{EE} / M_{\mathcal{L}}$, which can be expressed as

$$
\mathrm{EE}_{\mathrm{r}}=\frac{(1-\tau) \mathbb{E}\left[\log _{2}\left(1+\left(P_{\mathrm{r}}(\gamma)+\frac{e_{\mathrm{h}_{\mathrm{r}}} \tau}{1-\tau}\right) \gamma\right)\right]}{P_{\mathrm{cre}} \tau+(1-\tau)\left(P_{\mathrm{c}_{\mathrm{r}}}+\frac{1}{\varepsilon} \mathbb{E}_{\gamma}\left[P_{\mathrm{r}}(\gamma)\right]\right)} .
$$

Ratios of signal-to-noise, circuit-to-noise, harvest-to-noise power, and circuit-to-noise during harvesting are represented by $P_{\mathrm{r}}(\gamma)=P_{\mathrm{t}}(\gamma) / M_{\mathcal{L}}, P_{\mathrm{cr}}=P_{\mathrm{c}} / M_{\mathcal{L}}, e_{\mathrm{h}_{\mathrm{r}}}=e_{\mathrm{h}} / M_{\mathcal{L}}$, and $P_{\text {cre }}=P_{\mathrm{c}_{\mathrm{e}}} / M_{\mathcal{L}}$, respectively. Instead of joint maximization of

\footnotetext{
${ }^{1} P_{\mathrm{t}}(\gamma)$ is chosen such that it ensures the capacity at a given value of $\gamma$.

${ }^{2}$ The total harvested power is $\frac{e_{\mathrm{h}} \tau T_{\mathrm{S}}}{(1-\tau) T_{\mathrm{S}}}$, where $T_{\mathrm{S}}$ cancels out in (1).

${ }^{3}$ We note that effect of path- loss is included in $M_{\mathcal{L}}$.
} 
$\mathrm{EE}_{\mathrm{r}}$ and $\mathrm{SE}$, here, we minimize the inverse of the objective function in (4), to get

$$
\begin{aligned}
& \min _{P_{\mathrm{r}}(\gamma) \geq 0} \frac{1}{\mathrm{EE}_{\mathrm{r}}} \quad \text { and } \quad \min _{P_{\mathrm{r}}(\gamma) \geq 0} \frac{1}{\mathrm{SE}} \\
& \text { subject to: } \quad \mathbb{E}_{\gamma}\left[P_{\mathrm{r}}(\gamma)\right] \leq \frac{P_{\max }}{M_{\mathcal{L}}} .
\end{aligned}
$$

In order to solve the MOP in (7) and achieve pareto optimality of the solution, the MOP is converted into a SOP using a weighted sum method ${ }^{4}$ [19]. The objective function in (7) can hence be converted into,

$$
\begin{aligned}
& \min _{P_{\mathrm{r}}(\gamma) \geq 0} \frac{\Delta}{\mathrm{EE}_{\mathrm{r}}}+\frac{1-\Delta}{\mathrm{SE}} \\
& \text { subject to: } \quad \mathbb{E}_{\gamma}\left[P_{\mathrm{r}}(\gamma)\right] \leq \frac{P_{\max }}{M_{\mathcal{L}}},
\end{aligned}
$$

where $\Delta \in[0,1]$ is a weight factor that sets the priority of the two objective functions pertaining to $\mathrm{EE}_{\mathrm{r}}$ and $\mathrm{SE}$. The trade-off problem reduces to a SE-maximization problem when $\Delta=0$, and to an EE-maximization when $\Delta=1$. Here, varying $\Delta$ will decide the importance of $\mathrm{EE}_{\mathrm{r}}$ as $\Delta$ changes between 0 to 1 . Also, the $\mathrm{EE}_{\mathrm{r}}$ can be written in a ratio form as defined in (6), to get

$$
\min _{P_{\mathrm{r}}(\gamma) \geq 0} \frac{\Delta\left(P_{\mathrm{cre}} \tau+(1-\tau)\left(P_{\mathrm{c}_{\mathrm{r}}}+\frac{1}{\varepsilon} \mathbb{E}_{\gamma}\left[P_{\mathrm{r}}(\gamma)\right]\right)\right)+(1-\Delta)}{\mathrm{SE}}
$$

subject to: $\quad \mathbb{E}_{\gamma}\left[P_{\mathrm{r}}(\gamma)\right] \leq \frac{P_{\max }}{M_{\mathcal{L}}}$.

The formulation in (11) can be rewritten in a maximization form by inverting the objective function, and then replacing SE by its expression in (1). In this way, the optimization problem becomes

$$
\max _{P_{\mathrm{r}}(\gamma) \geq 0} \frac{(1-\tau) \mathbb{E}_{\gamma}\left[\log _{2}\left(1+\left(P_{\mathrm{r}}(\gamma)+\frac{e_{\mathrm{hr}} \tau}{1-\tau}\right) \gamma\right)\right]}{\Delta\left(P_{\mathrm{cre}} \tau+(1-\tau)\left(P_{\mathrm{c}_{\mathrm{r}}}+\frac{1}{\varepsilon} \mathbb{E}_{\gamma}\left[P_{\mathrm{r}}(\gamma)\right]\right)\right)+(1-\Delta)}
$$

subject to: $\quad \mathbb{E}_{\gamma}\left[P_{\mathrm{r}}(\gamma)\right] \leq \frac{P_{\max }}{M_{\mathcal{L}}}$.

\section{A. Optimal Power Allocation with No Input Power Constraint}

In this section, the unconstrained SOP is discussed to pave the way for the optimal power allocation strategy of the powerconstrained SOP. Hence, the unconstrained SOP is first solved for the optimum power allocation scheme with no input average power constraint. It is noted that the maximization problem in (13) involves maximization of a ratio of two functions of $P_{\mathrm{r}}(\gamma)$, and is not concave [20], [18]. However, the numerator of (13) is concave in the power, and the denominator is affine.

\footnotetext{
${ }^{4}$ Due to this scaling factor, $\mathrm{EE}_{\mathrm{r}}$ and $\mathrm{SE}$ are within same range. In order to make this summation meaningful, we assume that $\mathrm{EE}_{\mathrm{r}}$ is normalized by 1 bits $/ \mathrm{J} / \mathrm{Hz}$ and $\mathrm{SE}$ is normalized by $1 \mathrm{bits} / \mathrm{s} / \mathrm{Hz}$. However we do not include these normalization in equations for the sake of simplicity.
}

Hence, the EE-maximization function is strictly quasi-concave in $P_{\mathrm{r}}(\gamma)$, with a unique global maximum. In order to solve the quasi-concave optimization problem in (13)-(14), we use the fractional programming method [21].

Using the variable transformation with inverse power dissipation parameter for $\phi=$ $\Delta\left(P_{\mathrm{c}_{\mathrm{re}}} \tau+(1-\tau)\left(P_{\mathrm{c}_{\mathrm{r}}}+\frac{1}{\varepsilon} \mathbb{E}_{\gamma}\left[P_{\mathrm{r}}(\gamma)\right]\right)\right)+(1-\Delta), \quad$ the maximization problem in (13)-(14) is converted into

$\max \phi^{-1}\left((1-\tau) \mathbb{E}\left[\log _{2}\left(1+\left(P_{\mathrm{r}}(\gamma)+\frac{e_{\mathrm{h}_{\mathrm{r}}} \tau}{1-\tau}\right) \gamma\right)\right]\right)$

subject to:

$$
\begin{aligned}
& \phi^{-1}\left(\Delta\left(P_{\mathrm{c}_{\mathrm{re}}} \tau+(1-\tau)\left(P_{\mathrm{c}_{\mathrm{r}}}+\frac{1}{\varepsilon} \mathbb{E}_{\gamma}\left[P_{\mathrm{r}}(\gamma)\right]\right)\right)\right. \\
& \quad+(1-\Delta))=1, \\
& P_{\mathrm{r}}(\gamma) \geq 0 .
\end{aligned}
$$

The objective function in (15) is continuously differentiable, concave in $P_{\mathrm{r}}(\gamma)$, and the equality constraint is affine. Therefore, the Karush-Kuhn-Tucker (KKT) conditions are both sufficient and necessary for optimality of the solution [21]. Let $\hat{\lambda}$ be the Lagrangian multiplier, then the Lagrangian function will be given by

$$
\begin{aligned}
& L\left(P_{\mathrm{r}}(\gamma), \phi\right) \\
& =\phi^{-1}\left((1-\tau) \mathbb{E}_{\gamma}\left[\log _{2}\left(1+\left(P_{\mathrm{r}}(\gamma)+\frac{e_{\mathrm{h}_{\mathrm{r}}} \tau}{1-\tau}\right) \gamma\right)\right]\right) \\
& -\hat{\lambda}\left(\left(\phi ^ { - 1 } \left(\Delta\left(\tau P_{\mathrm{cre}}+(1-\tau) P_{\mathrm{cr}}+(1-\tau) \frac{1}{\varepsilon} \mathbb{E}_{\gamma}\left[P_{\mathrm{r}}(\gamma)\right]\right)\right.\right.\right. \\
& +(1-\Delta))-1))-\hat{\mu} P_{\mathrm{r}}(\gamma) .
\end{aligned}
$$

From (18), due to the complementary slackness, we have $\hat{\mu}=0$ when the strict inequality $P_{\mathrm{r}}(\gamma)>0$ holds. For optimal power allocation, the stationary conditions are written as,

$$
\begin{aligned}
& \frac{\partial L\left(P_{\mathrm{r}}(\gamma), \phi\right)}{\partial P_{\mathrm{r}}(\gamma)}=0, \\
& \frac{\partial L\left(P_{\mathrm{r}}(\gamma), \phi\right)}{\partial \phi}=0 .
\end{aligned}
$$

From (19), the optimum power allocation scheme can be derived as

$$
P_{\mathrm{r}}(\gamma)=\left[\frac{\varepsilon}{\hat{\lambda} \Delta}-\frac{1}{\gamma}-\frac{e_{\mathrm{h}_{\mathrm{r}}} \tau}{1-\tau}\right]^{+},
$$

where $[x]^{+}=\max (0, x)$.

We note that the power allocation (21) is a scaled and shifted version of traditional water-filling power allocation. This is due to the presence of the additional harvested power term, $\frac{e_{\mathrm{h}_{\mathrm{r}}} \tau}{1-\tau}$, and the time-switching parameter $\tau$. The expectation in (18) 
can be solved to find a closed-form expression for the optimal power strategy. We insert (21) into (20) and solve the closedform expressions for the expectation to obtain the value of $\hat{\lambda}$, which yields to

$$
\begin{aligned}
& (1-\tau) \log _{2}(\hat{\beta} \varepsilon) \mathrm{e}^{-\hat{\beta}}+(1-\tau) \operatorname{Ei}(\hat{\beta})-\left[(1-\tau) \mathrm{e}^{-\hat{\beta}}\right. \\
& \left.\log _{2}\left(\frac{\hat{\beta}(1-\tau)}{(1-\tau)+\hat{\beta} \tau e_{\mathrm{h}_{\mathrm{r}}}}\right)\right]-\frac{(1-\tau)^{2} \mathrm{e}^{-\hat{\beta}}}{\varepsilon\left((1-\tau)+\hat{\beta} \tau e_{\mathrm{h}_{\mathrm{r}}}\right)} \\
& -\frac{\hat{\beta}(1-\tau)(1-\Delta)}{\Delta\left((1-\tau)+\hat{\beta} \tau e_{\mathrm{h}_{\mathrm{r}}}\right)}-\frac{(1-\tau)^{2} P_{\mathrm{cr}} \hat{\beta}}{(1-\tau)+\hat{\beta} \tau e_{\mathrm{h}_{\mathrm{r}}}} \\
& +\frac{\hat{\beta}(1-\tau)^{2} \operatorname{Ei}(\hat{\beta})}{\varepsilon\left((1-\tau)+\hat{\beta} \tau e_{\mathrm{h}_{\mathrm{r}}}\right)}=0,
\end{aligned}
$$

where $\hat{\beta}=\frac{\Delta \hat{\lambda}(1-\tau)}{\varepsilon(1-\tau)-\tau \Delta e_{\mathrm{h}_{\mathrm{f}}} \hat{\lambda}}$, and $\operatorname{Ei}(\hat{\beta})=\int_{\hat{\beta}}^{\infty} \frac{\mathrm{e}^{-t}}{t} d t$ indicates the exponential integral [21]. Given that we provide the closed-form expressions, the optimization and computational complexity is very low.

\section{B. Optimal Power Allocation with Input Power Constraint}

Let us assume $\hat{\beta}^{*}$ is the optimal $\hat{\beta}$ that solves (22). The average input power at this point, $\bar{P}_{\mathrm{u}}$, can be found as $\bar{P}_{\mathrm{u}}=\left.\mathbb{E}_{\gamma}\left[P_{\mathrm{t}}(\gamma)\right]\right|_{\hat{\beta}=\hat{\beta}^{*}}$ from (21). Using $\bar{P}_{\mathrm{u}}$, one can show that the unconstrained EE-maximization problem can be simplified into a SE-maximization problem, subject to an input power constraint with the constraint power level set to $\bar{P}_{\mathrm{u}}$. Hence, the optimization problem in (13)-(14) gets simplified into a SE-maximization problem with average input power constraint given by $\mathbb{E}_{\gamma}\left[P_{\mathrm{r}}(\gamma)\right] \leq \min \left(\frac{\bar{P}_{\mathrm{u}}}{M_{\mathcal{L}}}, \frac{P_{\max }}{M_{\mathcal{L}}}\right)$.

\section{NumericAl Results}

In this section, simulation results are used to investigate the impact of the time-switching parameter $\tau$, the harvested power $e_{\mathrm{h}_{\mathrm{r}}}$, and the importance weight $\Delta$, on the achievable energy and spectral efficiency of the communication system. In all the provided plots, we assume that the power of the fixed battery at the transmitting device is represented by $P$. Unless otherwise stated, $\varepsilon$ is set to 0.2 and $\tau=0.5$ seeing similar parameters as in [18].

In Figure 1, we plot $\mathrm{EE}_{\mathrm{r}}$ versus the harvested power to the noise ratio $\left(e_{\mathrm{h}_{\mathrm{r}}}\right)$, when $P_{\mathrm{cr}_{\mathrm{r}}}=0 \mathrm{~dB}, P_{\mathrm{cre}}=0 \mathrm{~dB}$ and $\tau=0.5$, given various values of the importance weight $\Delta$. This figure is obtained from the theoretical result presented in (22). At lower values of $\Delta$, higher $\mathrm{EE}_{\mathrm{r}}$ can be achieved. In addition, higher $\mathrm{EE}_{\mathrm{r}}$ can be achieved at higher values of $e_{\mathrm{h}_{\mathrm{r}}}$, which shows that when the $\mathrm{EH}$ power is stronger, the system can harvest more energy during harvesting time, and in turn, achieves higher $\mathrm{EE}_{\mathrm{r}}$.

Similarly, Figure 2 shows the plots of SE versus $e_{\mathrm{h}_{\mathrm{r}}}$ for various values of the importance weight $\Delta$, with $P_{\mathrm{cr}}=0 \mathrm{~dB}$, $P_{\text {cre }}=0 \mathrm{~dB}$, and $\tau=0.5$. It is seen from the figure that SE monotonically increases with $e_{\mathrm{h}_{\mathrm{r}}}$ for lower values of the importance weight. When the importance weight is lower, i.e.,

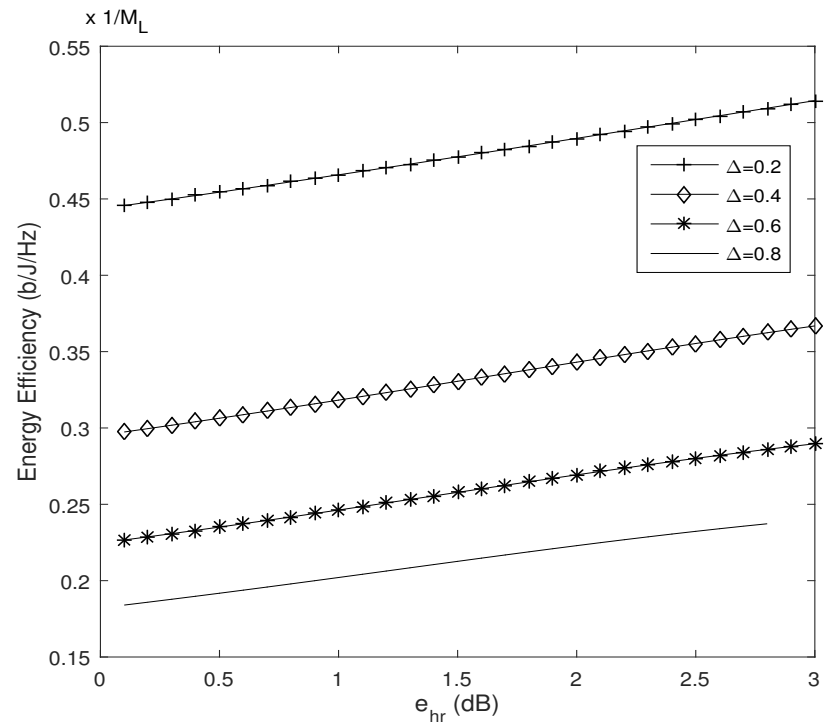

Figure 1. Energy efficiency versus the harvested power $e_{\mathrm{h}_{\mathrm{r}}}$, for various values of the importance weight $\Delta$, when $\tau=0.5, P_{\mathrm{c}_{\mathrm{r}}}=0 \mathrm{~dB}$ and $P_{\mathrm{cre}}=0 \mathrm{~dB}$.

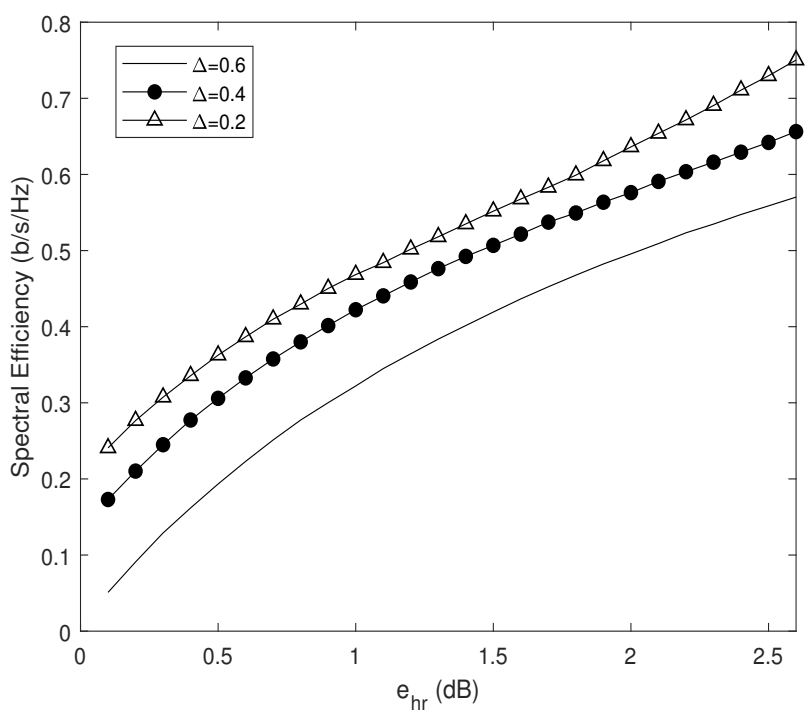

Figure 2. Spectral efficiency versus $e_{\mathrm{h}_{\mathrm{r}}}$ for various values of $\Delta$, when $\tau=0.5$, $P_{\mathrm{cr}}=0 \mathrm{~dB}$ and $P_{\mathrm{cre}}=0 \mathrm{~dB}$.

$\Delta=0.2$, SE increases slowly at lower values of the harvested power until it reaches $e_{\mathrm{h}_{\mathrm{r}}}=2 \mathrm{~dB}$. After that, a higher peak can be seen, which means that SE-maximization is dominant and confirms the significance of the importance weight in the trade-off given in (9).

Figure 3 shows $\mathrm{SE}$ versus $e_{\mathrm{h}_{\mathrm{r}}}$, when $\Delta=0.4, \tau=0.5$, $P_{\mathrm{cr}}=0 \mathrm{~dB}$ and $P_{\mathrm{cre}}=0 \mathrm{~dB}$. Different values of $\hat{\lambda}$ and $\hat{\beta}$ are considered. The figure depicts a monotonic increase in SE with respect to the harvested power. Also, the Lagrangian multiplier, $\hat{\lambda}$, decreases with increasing $e_{\mathrm{h}_{\mathrm{r}}}$, which can be confirmed from (21). $\hat{\beta}$, on the other hand, shows a very interesting behaviour: initially it increases to maximum value until a breakpoint, i.e., after which it decreases.

Figure 4 shows SE versus $\tau$ for different values of $\Delta$, when 


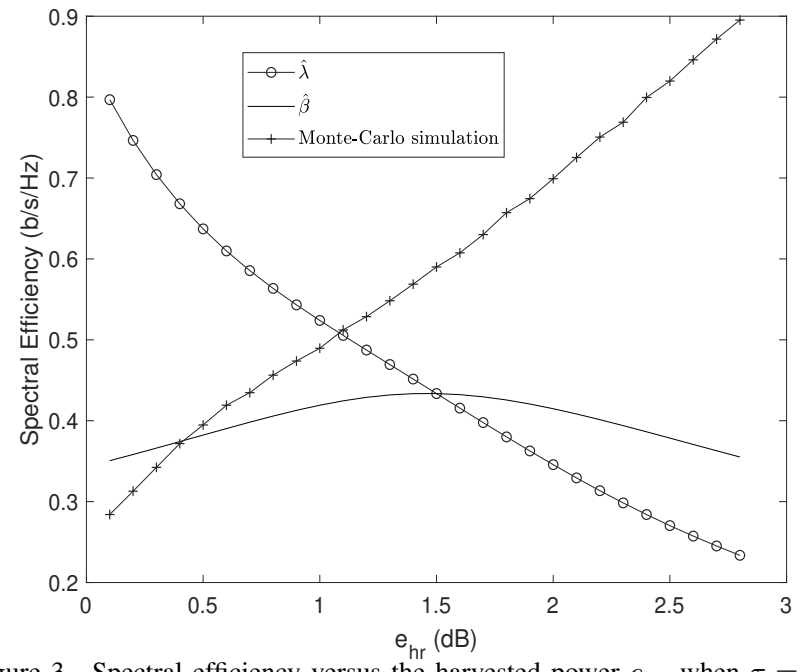

Figure 3. Spectral efficiency versus the harvested power $e_{\mathrm{h}_{\mathrm{r}}}$, when $\tau=0.5$, $\Delta=0.4, P_{\mathrm{cr}}=0 \mathrm{~dB}$ and $P_{\mathrm{cre}}=0 \mathrm{~dB}$ for different values of $\hat{\lambda}$ and $\hat{\beta}$.

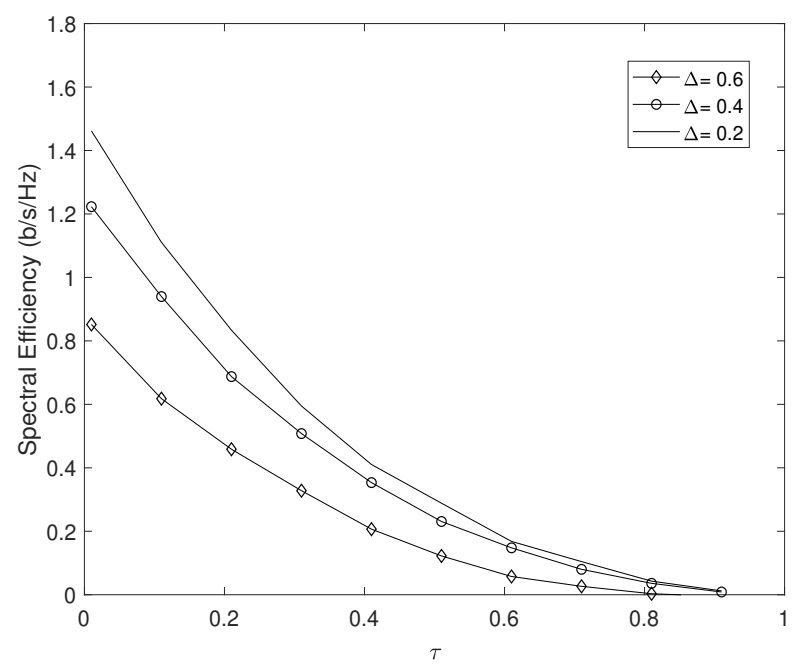

Figure 4. Spectral efficiency versus the time-switching parameter $\tau$ for different values of $\Delta$, with $e_{\mathrm{h}_{\mathrm{r}}}=0.5 \mathrm{~dB}, P_{\mathrm{cr}_{\mathrm{r}}}=0 \mathrm{~dB}$ and $P_{\text {cre }}=0 \mathrm{~dB}$.

$e_{\mathrm{h}_{\mathrm{r}}}=0.5 \mathrm{~dB}, P_{\mathrm{cr}}=0 \mathrm{~dB}$ and $P_{\mathrm{cre}}=0 \mathrm{~dB}$. It is noted that at $\tau=0$ and lower values of the importance weight $\Delta$, maximum SE can be achieved. Spectral efficiency is always monotonically decreasing with respect to all the values of $\Delta$. This confirms the behaviour of optimum power allocation scheme presented in (21). In the current setting, SE is monotonically decreasing with $\tau$ as well, which shows that less time should be spent for harvesting energy. This happens since $e_{\mathrm{h}_{\mathrm{r}}}$ is much smaller than the input power from the fixed battery.

Figure 5 shows $\mathrm{EE}_{\mathrm{r}}$ versus the time-switching parameter $\tau$ for various values of $P_{\mathrm{cr}}$ and $P_{\mathrm{cre}}$, with $\Delta=0.7$ and $e_{\mathrm{h}_{\mathrm{r}}}=1 \mathrm{~dB}$. It is noted that when $P_{\mathrm{cr}}=0 \mathrm{~dB}$ and $P_{\text {cre }}=0 \mathrm{~dB}$, maximum $\mathrm{EE}_{\mathrm{r}}$ is achieved at $\tau \approx 0.7$. After reaching its peak value, $\mathrm{EE}_{\mathrm{r}}$ decreases with the time-switching parameter. Also, when $P_{\mathrm{cr}}>P_{\mathrm{cre}}, \mathrm{EE}_{\mathrm{r}}$ increases continuously due to the fact that the allocation strategy for EE-maximization has consumed the

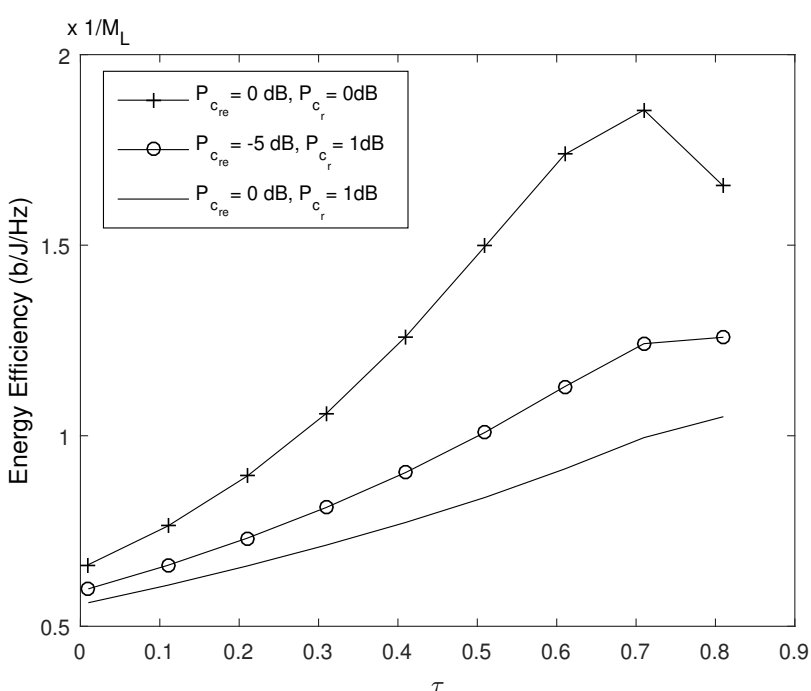

Figure 5. Energy efficiency versus the time-switching parameter $\tau$ for different values of $P_{\mathrm{c}_{\mathrm{r}}}$ and $P_{\mathrm{cre}}$, with $\Delta=0.7$ and $e_{h_{\mathrm{r}}}=1 \mathrm{~dB}$.

whole input power.

\section{CONCLUSION}

In this paper, an optimal power allocation scheme for the joint optimization of the energy efficiency (EE) and spectral efficiency (SE) of a point-to-point communication system equipped with fixed as well as energy harvesting batteries was obtained. In the considered system, the transmitter switches between harvesting energy and transmitting information according to a time-switching approach. A multi-objective optimization problem (MOP) to jointly optimize EE and SE was formulated, and an importance weight factor was introduced to convert the MOP into a single-objective optimization problem. The weight factor sets the priority level of EE versus SE. By using fractional programming and KKT conditions, the optimum power allocation scheme was first calculated when no input power constraint exists. The solution was then generalized for a system with input power constraint. Closed-form expressions for the power allocation scheme were derived. Monte-Carlo simulations were done to see the effect of the harvested power in improving the overall rate of the system. Numerical results were presented to analyze the impact of the importance weight with a system equipped with solar energy harvesting (EH) on the achievable trade-off performance. The system model proposed here can be used as a benchmark for more complex EH-MOP systems which deal with power allocation schemes for the joint optimization of EE and SE.

\section{REFERENCES}

[1] C. Yang, J. Li, M. Guizani, A. Anpalagan, and M. Elkashlan, "Advanced spectrum sharing in 5G cognitive heterogeneous networks," IEEE Wireless Commun., vol. 23, no. 2, pp. 94-101, Apr. 2016.

[2] H. Ostaffe, "RF-based wireless charging and energy harvesting enables new applications and improves product design," Powercast Corporation, 2017.

[3] D. Cai, J. Wu, J. Yang, and P. Fan, "Optimum spectral and energy efficiency tradeoff in two-user Gaussian interference channel," IEEE Wireless Commun. Let., vol. 7, no. 3, pp. 468-471, Jun. 2018. 
[4] S. Zhang, N. Zhang, G. Kang, and Z. Liu, "Energy and spectrum efficient power allocation with NOMA in downlink hetnets," Physical Communication, vol. 31, pp. 121-132, Dec. 2018.

[5] L. Deng, Y. Rui, P. Cheng, J. Zhang, Q. Zhang, and M. Li, "A unified energy efficiency and spectral efficiency tradeoff metric in wireless networks," IEEE Commun. Let., vol. 17, no. 1, pp. 55-58, Jan. 2013.

[6] F. Benkhelifa, K. Tourki, and M.-S. Alouini, "Simultaneous wireless information and power transfer for spectrum sharing in cognitive radio communication systems," in Proc. Int. Conf. Commun., (ICC), Malaysia, May 2016, pp. 676-681.

[7] A. M. Siddiqui, L. Musavian, S. Aissa, and Q. Ni, "Performance analysis of relaying systems with fixed and energy harvesting batteries," IEEE Trans. on Commun., vol. 66, no. 4, pp. 1386-1398, Apr. 2018.

[8] J. Tang, D. K. So, E. Alsusa, and K. A. Hamdi, "Resource efficiency: A new paradigm on energy efficiency and spectral efficiency tradeoff," IEEE Trans. Wireless Commun., vol. 13, no. 8, pp. 4656-4669, Aug. 2014.

[9] Y. Gao, H. He, Z. Deng, and X. Zhang, "Cognitive radio network with energy-harvesting based on primary and secondary user signals," IEEE Access, vol. 6, pp. 9081-9090, Feb. 2018.

[10] Q. Wang, H.-L. Liu, J. Yuan, and L. Chen, "Optimizing the energyspectrum efficiency of cellular systems by evolutionary multi-objective algorithm," Integrated Comp. Aided Engin., vol. 26, no. 2, pp. 207-220, Jan. 2019.

[11] O. Amin, E. Bedeer, M. H. Ahmed, and O. A. Dobre, "Energy efficiency and spectral efficiency trade-off for OFDM systems with imperfect channel estimation," in Proc. IEEE Int. Conf. Commun. (ICC), Australia, Jun. 2014, pp. 3553-3558.

[12] D. Ni, L. Hao, X. Qian, and Q. T. Tran, "Energy-spectral efficiency tradeoff of downlink NOMA system with fairness consideration," in in Proc. IEEE 87th Veh. Techn. Conf. (VTC), Chicago, Jun. 2018, pp. 1-5.

[13] M. R. Mili and L. Musavian, "Interference efficiency: A new metric to analyze the performance of cognitive radio networks." IEEE Trans. Wireless Commun., vol. 16, no. 4, pp. 2123-2138, Jan. 2017.

[14] B. Huang and A. Guo, "Optimal design of energy-and spectral-efficient wireless backhaul hetnets: Joint load balancing and interference management," IEEE Access, vol. 6, pp. 68 450-68 462, 2018.

[15] J. Sun, W. Zhang, J. Sun, C.-X. Wang, and Y.-F. Chen, "Energy-spectral efficiency in simultaneous wireless information and power transfer," in Proc. IEEE Conf. Commun. (ICCC), China, Jul. 2016, pp. 1-6.

[16] R. Zhang, Y. Li, C.-X. Wang, Y. Ruan, and H. Zhang, "Performance tradeoff in relay aided D2D-cellular networks," IEEE Trans. Vehic. Tech., Jul. 2018.

[17] Y. Liu, S. A. Mousavifar, Y. Deng, C. Leung, and M. Elkashlan, "Wireless energy harvesting in a cognitive relay network," IEEE Trans. Wireless Commun., vol. 15, no. 4, pp. 2498-2508, 2016.

[18] A. M. Siddiqui, L. Musavian, and Q. Ni, "Energy efficiency optimization with energy harvesting using harvest-use approach," in Proc. IEEE Int. Conf. Commun. Workshop (ICCW)., London, Jun. 2015, pp. 1982-1987.

[19] W. Yu, L. Musavian, and Q. Ni, "Tradeoff analysis and joint optimization of link-layer energy efficiency and effective capacity toward green communications," IEEE Trans. Wireless Commun., vol. 15, no. 5, pp. 3339-3353, May 2016.

[20] S. Schaible, "Minimization of ratios," J. of Optimization Theory and Applic., vol. 19, no. 2, pp. 347-352, Jun. 1976.

[21] S. Boyd and L. Vandenberghe, "Convex optimization. 2004," Cambridge Univ. Pr, 2004 